\title{
Tissue-Specific Splicing
}

National Cancer Institute

\section{Source}

National Cancer Institute. Tissue-Specific Splicing. NCI Thesaurus. Code C19564.

Alternate splicing which is specific and typical for a given tissue. 